\title{
Étude pilote sur l'utilité et l'applicabilité d'un outil de plan de soins pharmaceutiques au Québec et en France
}

\author{
Pilot study on the usefulness and the application of a pharmaceutical care \\ plan tool in Quebec and France
}

Jean-François BUSSIÈRES ${ }^{1}$, Ariane BLANC ${ }^{2}$, Annie LAVOIE ${ }^{1}$, Josiane GAGNON-BAGHERI ${ }^{1}$ et André RIEUTORD ${ }^{2}$

1 Unité de recherche en pratique pharmaceutique, Département de Pharmacie, Centre hospitalier universitaire SainteJustine, Université de Montréal, 3175 chemin de la côte Sainte-Catherine, Montréal, H3T 1C5, Canada.

2 Service de pharmacie, Hôpital Robert Debré, APHP, 48 Bd Sérurier, 75935 Paris Cedex 19, France.

Manuscrit reçu le 30 mai 2008 ; commentaires éditoriaux formulés aux auteurs le 5 août 2008 ; accepté pour publication le 8 septembre 2008

Mots clés :

Soins pharmaceutiques ;

plan de soins ;

visioconference ;

pharmacie clinique

Keywords:

Pharmaceutical care;

care plan;

visioconference;

clinical pharmacy
Résumé - Contexte : Le plan de soins pharmaceutiques est un outil particulièrement développé en Amérique du Nord. Il a pour but de prévenir, identifier ou résoudre les problèmes liés aux produits de santé du patient. But : Cette étude pilote avait pour objectif de décrire l'utilité et l'applicabilité d'un plan de soins pharmaceutiques dans le cadre de formation simultanément au Québec et en France. Méthode : Quatre pharmaciens tuteurs supervisaient 8 étudiants en pharmacie québécois et français qui débutaient leur stage clinique en milieu hospitalier. Les étudiants disposaient de quatre semaines pour colliger les données sur leurs patients et rédiger deux plans de soins pharmaceutiques. À la fin de cette période, une visioconférence Québec-France fut organisée afin d'échanger sur les seize plans de soins. Résultats : L'outil a été plus apprécié par les étudiants français peu rompus à l'approche de soins pharmaceutiques. La visioconférence organisée entre les deux groupes d'étudiants a montré des différences d'évaluation des étudiants par les tuteurs plus interventionnistes ou centrés sur la pathologie pour les québécois et français respectivement. Conclusion : Ce projet a mis en évidence l'intérêt de l'outil pour le plan de soins et sa transposition possible dans le contexte français. Enfin, la visioconférence pourrait permettre de développer le concept de soins pharmaceutiques dans les hôpitaux ou les universités où les formateurs compétents font défaut.

Abstract - Context: The pharmaceutical care plan is a tool particularly developed in North America. It is designed to prevent, identify or solve problems related to patient health products. Objective: The aim of this study was to describe the application and the utility of a pharmaceutical care plan in the context of a simultaneous formation in Quebec and France. Method: Four pharmacists supervised eight pharmacy students who started their hospital clinical internship. Students had four weeks to collect patient information and complete two pharmaceutical care plans. At the end of this period, a visioconference was organised in Quebec and France in order to discuss about the 16 pharmaceutical care plans. Results: This tool was more appreciated by the French students who were less familiar with the care approach. The visioconference has showed differences regarding student assessment 
pharmaceutical by tutors, which were either more interventionist or centered on the pathology in Quebec and France, respectively. Conclusion: This project highlighted the interest of the care plan tool and its possible transferability in a French context. Finally, the visioconference could allow the development of the pharmaceutical care concept in hospitals or universities, where the competency of trainers are deficient.

\section{Introduction}

Depuis l'identification et la description du concept de soins pharmaceutiques en 1990 par Hepler et Strand ${ }^{[1]}$, les programmes de formation universitaire de pharmacie en Amérique du Nord ont intégré ce concept aux approches pédagogiques. L'évolution du rôle clinique du pharmacien a contribué à la transformation des programmes de baccalauréat en pharmacie (B. Pharm.) en programmes de doctorat professionnel (Pharm. D.) à l'échelle des États-Unis au début des années deux mille et depuis septembre 2007 dans une faculté de pharmacie au Canada (université de Montréal).

En 1998, la fédération internationale pharmaceutique (FIP) a publié une déclaration sur les normes professionnelles et l'application des soins pharmaceutiques ${ }^{[2]}$. Elle définit les soins pharmaceutiques comme étant : «les dispositions responsables de la pharmacothérapie ayant pour but d'obtenir des résultats définis qui améliorent ou maintiennent la qualité de vie d'un malade. C'est un processus de collaboration qui a pour but de prévenir ou d'identifier et de résoudre les problèmes reliés aux produits de santé. Ceci est un processus continuel d'amélioration de la qualité en ce qui concerne l'emploi de produits de santé ».

L'évolution de la pharmacie clinique et de l'intégration du concept de soins pharmaceutiques peut être retracée à partir d'une série d'articles publiés dans Annals of Pharmacotherapy depuis 2005 en pratique communautaire, notamment au Canada $^{[3]}$, aux États-Unis ${ }^{[4]}$ au Royaume-Uni ${ }^{[5]}$, en Allemagne ${ }^{[6]}$ et en Suisse ${ }^{[7]}$. De nombreux articles ont été aussi publiés sur le rôle clinique du pharmacien hospitalier.

Afin d'intégrer le concept de soins pharmaceutiques à l'enseignement théorique et pratique, les professeurs et les pharmaciens cliniciens ont développé des outils afin de former les étudiants et de soutenir la démarche de soins pharmaceutiques. Le terme plan de soins, introduit en 1968, a d'abord été défini comme « un plan de soins médicaux ou infirmiers, généralement sous forme écrite, rédigé pour un patient en particulier ». Bien que le plan de soins n'était a priori pas un outil destiné aux pharmaciens, il fait désormais partie de leur pratique dans plusieurs pays. En croisant les termes patient care planning et pharmacy, sont dénombrés 157 articles dans les bases de données bibliographiques de la National Library of Medicine.

Cradock et al. décrivent dès 1972 la pertinence pour un pharmacien de documenter des aspects cliniques de l'état de santé du patient ${ }^{[8]}$. La même année, Cooper et al. identifient des critères afin d'aider le pharmacien à sélectionner les patients nécessitant un suivi clinique ${ }^{[9]}$. Bennett et al. décrivent pour la première fois en 1973 le concept de plan de soins du pharmacien ${ }^{[10]}$. Il faut toutefois attendre deux décennies avant que le concept d'un plan de soins en lien avec le concept de soins pharmaceutiques ne soit décrit.

En 1993-1994, plusieurs auteurs documentent le recours à des plans de soins pharmaceutiques, tant aux États-Unis ${ }^{[11,12]}$ qu'au Canada ${ }^{[13]}$. Dès 1994, la Joint Commission on Accreditation of Healthcare Organizations intègre aux normes d'agrément des hôpitaux le concept de plan de soins pharmaceutiques $^{[14]}$.

En France, celui-ci est encore balbutiant; il est intégré dans la pratique clinique dans quelques hôpitaux précurseurs ou pratiqué, comme à l'hôpital Robert Debré (Paris), uniquement dans le cadre de l'enseignement. 
L'hôpital de Sainte-Justine à Montréal et l'hôpital Robert Debré à Paris étant jumelés, il nous a paru intéressant de confronter nos pratiques sur le plan de soins pharmaceutiques. Il s'agit d'une étude pilote observationnelle et descriptive réalisée en collaboration avec les centres hospitaliers universitaires mèreenfant à Montréal (CHU Sainte-Justine) et à Paris (hôpital Robert Debré). L'objectif de l'étude est de déterminer l'utilité et l'applicabilité d'un outil de plan de soins pharmaceutiques dans le cadre du programme de formation en pharmacie simultanément au Québec et en France.

\section{Collectifs et méthodes}

\section{Population}

Quatre étudiants en quatrième année du programme de premier cycle de la faculté de pharmacie de l'université de Montréal et quatre étudiants de la faculté de pharmacie de l'université Paris V-René Descartes en cinquième année hospitalo-universitaire ont été recrutés. Ceux-ci effectuaient un stage clinique hospitalier d'une durée de sept et dix-sept semaines à Montréal et à Paris. Les étudiants ont tous débuté leurs stages en même temps et étaient «naïfs » quant aux services choisis. Les services cliniques choisis étaient la pédiatrie générale (deux étudiants) et la réanimation (deux étudiants) à Robert Debré et Sainte-Justine simultanément. Les quatre pharmaciens tuteurs (trois québécois, un français) avaient une expérience de l'approche du Pharmaceutical Care (théorique et pratique) d'au moins cinq ans avec une moyenne de 13 ans pour l'ensemble de l'équipe. Tous les membres enseignaient la pharmacie clinique dans un cadre soit universitaire, soit hospitalier ou encore dans le cadre de séminaires de formation continue. Deux pharmaciens étaient basés à Montréal, deux autres à Paris. L'étude s'est déroulée durant six semaines de janvier à mars 2008.

\section{Intervention}

Compte tenu que l'outil de plan de soins pharmaceutiques est en place à la faculté de pharmacie de l'université de Montréal depuis 1994 et qu'il est connu des étudiants québécois et utilisé dans le cadre de la formation avant la tenue de stages hospitaliers, l'équipe de recherche a développé un guide de formation à l'intention des étudiants et diffusé le guide à tous les étudiants recrutés. L'outil «plan de soins pharmaceutiques » utilisé pour cette étude pilote est un document d'une page recto-verso. Le recto contient les données démographiques du patient, ses allergies et intolérances, les problèmes à l'admission, les diagnostics et antécédents médicaux, les médicaments utilisés auparavant ainsi que l'histoire pharmacologique active et les tests de laboratoire et examens de suivis pertinents. Le verso contient une grille pour le suivi de trois à six problèmes reliés à la pharmacothérapie. Pour chaque problème relié à la pharmacothérapie, l'étudiant doit décrire les résultats recherchés, les solutions possibles, la solution retenue, les interventions à effectuer, le monitorage et sa fréquence et les résultats obtenus lors du suivi.

Le guide de formation de 14 pages comprend une description du concept de soins pharmaceutiques. À ce guide, sont joints des consignes relatives à l'utilisation de plans de soins pharmaceutiques, quatre exemples de plans rédigés par des étudiants québécois à l'automne 2007 et le gabarit vierge de plans de soins pharmaceutiques. Le guide de formation a été diffusé par courriel aux huit étudiants recrutés une semaine avant le début de l'étude. Les étudiants ont pu formuler par courriel des questions quant au contenu du guide de formation et du projet de recherche. La formation, les objectifs et le déroulement séquentiel de l'étude ont été délivrés localement par les pharmaciens tuteurs respectifs.

L'outil de plan de soins pharmaceutiques a pour objectif de structurer la collecte de données, l'identification de problèmes reliés à la pharmacothérapie et de structurer la démarche de résolution de ces problèmes. Dans le cadre du concept de soins pharmaceutiques, le pharmacien participe activement à la résolution du problème et assure le suivi des résultats recherchés.

Après avoir achevé la formation (deux semaines), les étudiants ont été invités à utiliser l'outil de plan de soins pharmaceutiques pour les patients 
dont ils assument la responsabilité durant le stage clinique de pharmacie dans les unités de soins de pédiatrie de chaque hôpital. Sur une période de deux semaines, chaque étudiant devait identifier au moins deux cas de patients et utiliser l'outil de plan de soins pharmaceutiques pour en assurer le suivi. Les étudiants de chaque hôpital étaient encadrés par deux pharmaciens.

Au terme d'une période de collecte de données de deux semaines, les étudiants ont été invités à échanger par courriel les seize plans de soins anonymisés et informatisés auprès des étudiants de l'autre pays. Après avoir pris connaissance des plans de soins des autres étudiants, chaque étudiant a été invité à répondre au questionnaire d'évaluation. Par la suite, une visioconférence QuébecFrance de deux heures et demie a été organisée. Durant la visioconférence, l'équipe de recherche a fait le point sur le projet, puis procédé à une simulation pharmacien-étudiant du modèle académique d'évaluation en ayant recours au plan de soins pharmaceutiques. Chaque étudiant québécois a été sollicité pour présenter en six à huit minutes son plan de soins. À l'issue de sa présentation, le pharmacien tuteur québécois a questionné individuellement chacun des quatre étudiants québécois pendant une durée de six à huit minutes. Cet entretien avait pour but de vérifier sa compréhension, sa maîtrise de l'un des deux cas et sa capacité à intervenir dans une démarche de soins au sein de l'équipe médicale et paramédicale. Les questions étaient ouvertes, suggestives, adaptées au cas cliniques, non standardisées. Elles visaient à renforcer voire compléter le plan de soins pharmaceutiques présenté et à stimuler la réflexion de l'étudiant dans le contexte de plan de soins pharmaceutiques. La même démarche a été menée par l'équipe française. Au terme des deux simulations, l'ensemble des participants (quatre pharmaciens, huit étudiants) a échangé sur la démarche.

\section{Critères d'analyses}

Les critères primaires de l'étude sont la mesure de la perception de l'utilité et de l'applicabilité du plan de soins pharmaceutiques par les étudiants. Les critères secondaires incluent la description du déroulement de la simulation en visioconférence et le recueil des observations des participants (c'est-à-dire pharmaciens et étudiants) durant la simulation. Il faut noter, qu'en aucun cas, la qualité et la quantité du travail remis par chaque étudiant n'étaient jugées dans le cadre de cette étude.

\section{Outils}

L'évaluation de la perception de l'utilité et de l'applicabilité du plan de soins pharmaceutiques a été effectuée à l'aide d'un questionnaire de 10 questions avec une échelle de Likert à quatre items (tout à fait d'accord, partiellement en accord, partiellement en désaccord, tout à fait en désaccord). Le questionnaire a été pré-testé auprès de quatre étudiants (deux étudiants québécois et deux étudiants français) afin de vérifier la clarté des énoncés. Des modifications mineures ont été apportées à la formulation de trois énoncés.

\section{Analyse}

Le niveau d'accord aux énoncés est présenté sans analyse statistique compte tenu du caractère pilote et descriptif de l'étude et du faible effectif d'observation.

\section{Résultats}

Le tableau I présente le portrait démographique des huit étudiants recrutés, soit quatre étudiants québécois et quatre étudiants français. Aucun étudiant n'a refusé de participer à l'étude. Il s'agit d'un échantillon non homogène d'étudiants ayant une exposition préalable différente à la pratique en officine et en hôpital. Le temps consacré à la collecte des données et à la complétion des plans de soins pharmaceutiques varie entre les étudiants (de deux à 
Tableau I. Caractéristiques des étudiants recrutés dans la pratique de l'exercice pédagogique des plans de soins pharmaceutiques.

\begin{tabular}{llllllllll}
\hline Variables & Unité & \multicolumn{2}{l}{ Étudiants québécois } & \multicolumn{5}{c}{ Étudiants français } \\
\hline Âge & Années & 26 & 28 & 28 & 34 & 22 & 22 & 23 & 23 \\
\hline Sexe & M, F & F & M & F & F & F & M & F & F \\
\hline $\begin{array}{l}\text { Exposition à la } \\
\text { pratique en officine }\end{array}$ & oui/non & Oui & Oui & Oui & Oui & Non & Oui & Oui & Oui \\
\hline $\begin{array}{l}\text { Durée de } \\
\text { l'exposition }\end{array}$ & A, B, C & C & B & C & B & NA & C & B & C \\
\hline $\begin{array}{l}\text { Exposition à la } \\
\text { pratique en hôpital }\end{array}$ & oui/non & Oui & Oui & Non & Oui & Non & Non & Oui & Non \\
\hline $\begin{array}{l}\text { Durée de } \\
\text { l'exposition }\end{array}$ & A, B, C & C & C & NA & C & NA & NA & C & NA \\
\hline $\begin{array}{l}\text { Temps requis pour } \\
\text { colliger les données } \\
\times 2 \text { patients }\end{array}$ & Heures & 3 & 8 & 6 & 2 & 12 & 2 & 2 & 8 \\
\hline $\begin{array}{l}\text { Temps requis pour } \\
\text { compléter les plans } \\
\text { de } \\
\text { soins } \times 2 \text { patients }\end{array}$ & Heures & 4 & 4 & 8 & 4 & 8 & 2 & 3 & 6 \\
\hline
\end{tabular}

Durée d'exposition : A) moins de 30 jours, B) 31-100 jours, C) >100 jours

$\mathrm{NA}=$ non applicable, $\mathrm{M}=$ masculin, $\mathrm{F}=$ féminin.

huit heures pour les étudiants québécois et de deux à douze pour les étudiants français).

Le tableau II présente la perception des étudiants quant à l'utilité et l'applicabilité de l'outil de plans de soins pharmaceutiques. De façon générale, les étudiants québécois et français ont un niveau d'accord similaire pour la plupart des énoncés. Toutefois, les étudiants québécois considèrent l'outil moins applicable que les étudiants français à leur pratique professionnelle en dehors du cadre d'enseignement en officine ou en hôpital. Dans le cadre de l'évaluation, les étudiants ont formulé des suggestions quant à la l'amélioration de l'outil, notamment grâce à un espace dédié aux conditions de délivrance de médicaments (c'est-à-dire médicaments sans avis de conformité/autorisation de mise en marché) et aux conditions d'administration des médicaments. Selon la nature des patients suivis, les étudiants ont suggéré d'ajuster l'espace disponible pour la documentation de médicaments, de tests de laboratoire ou de particularités. De façon générale, les étudiants mentionnent que l'outil est très pertinent.

Lors de la simulation pharmacien-étudiant, il est intéressant de noter parmi les verbatim recueillis quelques similarités et quelques différences. La pharmacienne du groupe Québec discutait avec les étudiants en utilisant des questions telles que : «Qu'est-ce que tu en penses? Est-ce ton choix? Que proposes-tu comme suivi? Que dirais-tu à la patiente? Est-ce que tu es d'accord avec ça? Sur 
Tableau II. Perception des étudiants de l'utilité et de l'applicabilité de l'outil de plans de soins pharmaceutiques.

\begin{tabular}{|c|c|c|c|c|c|c|c|c|}
\hline \multirow[t]{2}{*}{ L'outil de plan de soins ... } & \multicolumn{4}{|c|}{ Étudiants québécois } & \multicolumn{4}{|c|}{ Étudiants français } \\
\hline & 1 & 2 & 3 & 4 & 1 & 2 & 3 & 4 \\
\hline 1. Est facile à utiliser & $\mathrm{a}$ & $\mathrm{a}$ & $\mathrm{b}$ & $\mathrm{a}$ & $\mathrm{b}$ & a & $\mathrm{b}$ & $\mathrm{a}$ \\
\hline \multicolumn{9}{|l|}{ 2. Inclut tous les renseignements pertinents } \\
\hline à recueillir & $\mathrm{a}$ & $\mathrm{a}$ & $\mathrm{b}$ & $\mathrm{a}$ & $\mathrm{b}$ & $\mathrm{b}$ & $\mathrm{b}$ & $\mathrm{b}$ \\
\hline 3. Est pratique pour participer aux activités cliniques dans les services & $\mathrm{a}$ & $\mathrm{b}$ & a & $\mathrm{a}$ & $\mathrm{a}$ & $\mathrm{a}$ & $\mathrm{a}$ & $\mathrm{a}$ \\
\hline $\begin{array}{l}\text { 4. Est adéquat pour permettre une documentation complète } \\
\text { des différents éléments }\end{array}$ & $\mathrm{a}$ & $\mathrm{b}$ & $\mathrm{b}$ & $\mathrm{b}$ & $\mathrm{c}$ & $\mathrm{a}$ & $\mathrm{a}$ & $\mathrm{b}$ \\
\hline 5. Est un bon outil de suivi quotidien des patients & $\mathrm{a}$ & a & a & $a$ & $\mathrm{~b}$ & $\mathrm{~b}$ & $\mathrm{a}$ & $\mathrm{a}$ \\
\hline $\begin{array}{l}\text { 6. Permet d'appliquer la démarche des soins pharmaceutiques } \\
\text { qui repose sur l'identification de problèmes reliés à la } \\
\text { pharmacothérapie (PRP) et de solutions }\end{array}$ & a & $\mathrm{a}$ & $\mathrm{a}$ & $\mathrm{a}$ & $\mathrm{a}$ & $\mathrm{a}$ & $\mathrm{b}$ & $\mathrm{a}$ \\
\hline $\begin{array}{l}\text { 7. Permet d'effectuer efficacement le suivi des PRP, } \\
\text { des solutions et des interventions }\end{array}$ & $\mathrm{a}$ & $\mathrm{b}$ & $\mathrm{a}$ & $\mathrm{a}$ & $\mathrm{a}$ & $\mathrm{a}$ & $\mathrm{b}$ & $\mathrm{b}$ \\
\hline $\begin{array}{l}\text { 8. Devrait être utilisé dans le cadre } \\
\text { des stages de } 1^{\mathrm{er}} \text { cycle (i.e. } 4^{\mathrm{e}} \text { année ou } 5^{\mathrm{e}} \text { AHU) }\end{array}$ & $\mathrm{a}$ & $\mathrm{a}$ & $\mathrm{a}$ & $\mathrm{a}$ & $\mathrm{a}$ & $\mathrm{a}$ & $\mathrm{a}$ & $\mathrm{a}$ \\
\hline $\begin{array}{l}\text { 9. Devrait être utilisé dans ma pratique professionnelle en } \\
\text { dehors du cadre d'enseignement en officine }\end{array}$ & $\mathrm{c}$ & $\mathrm{b}$ & $\mathrm{b}$ & $\mathrm{b}$ & $\mathrm{c}$ & $\mathrm{a}$ & $\mathrm{a}$ & $\mathrm{a}$ \\
\hline $\begin{array}{l}\text { 10. Devrait être utilisé dans ma pratique professionnelle } \\
\text { en dehors du cadre d'enseignement en hôpital }\end{array}$ & $\mathrm{b}$ & $b$ & $\mathrm{~b}$ & $\mathrm{a}$ & $\mathrm{a}$ & $\mathrm{a}$ & $\mathrm{c}$ & $\mathrm{d}$ \\
\hline
\end{tabular}

Échelle de réponse - a - très en accord, b - partiellement en accord, $\mathrm{c}$ - partiellement en désaccord, $\mathrm{d}$ - totalement en désaccord. $\mathrm{AHU}=$ année hospital universitaire.

quoi te bases-tu pour affirmer que... ?». La pharmacienne du groupe France discutait avec les étudiants en utilisant des questions telles que : «Que cherche-t-on comme signes et symptômes pour un patient de ce type ? Est-ce que tu connais les recommandations? Quel est le rôle du pharmacien dans un conseil ? Qu'est-ce qui pourrait être surveillé ? De façon générale, que doit-on faire pour cette pathologie? ».

Le tableau III présente un exemple d'un plan de soins pharmaceutiques détaillé réalisé par une étudiante québécoise. L'étudiante a identifié six problèmes reliés à la pharmacothérapie. Trois de ces problèmes ont été identifiés et discutés selon l'outil. Par ailleurs, l'étudiant a aussi documenté et proposé ses stratégies pour les trois autres problèmes suivants, non décrits dans le tableau, à savoir le retard de croissance secondaire à une maladie chronique (mucoviscidose), la présence de polypes nasaux et la nécessité d'une prophylaxie contre les surinfections pulmonaires à Staphylococcus aureus lors des rhumes et grippes. Le texte du tableau n'a pas été modifié afin de présenter l'utilisation réelle de l'outil et les annotations requises dans le cadre académique et clinique.

\section{Discussion}

Bien que le concept de soins pharmaceutiques ait presque deux décennies, son implantation varie d'un pays à l'autre. Alors qu'au Québec les programmes universitaires de formation en pharmacie ont intégré 
Tableau III. Exemple d'un plan de soins pharmaceutiques détaillé.

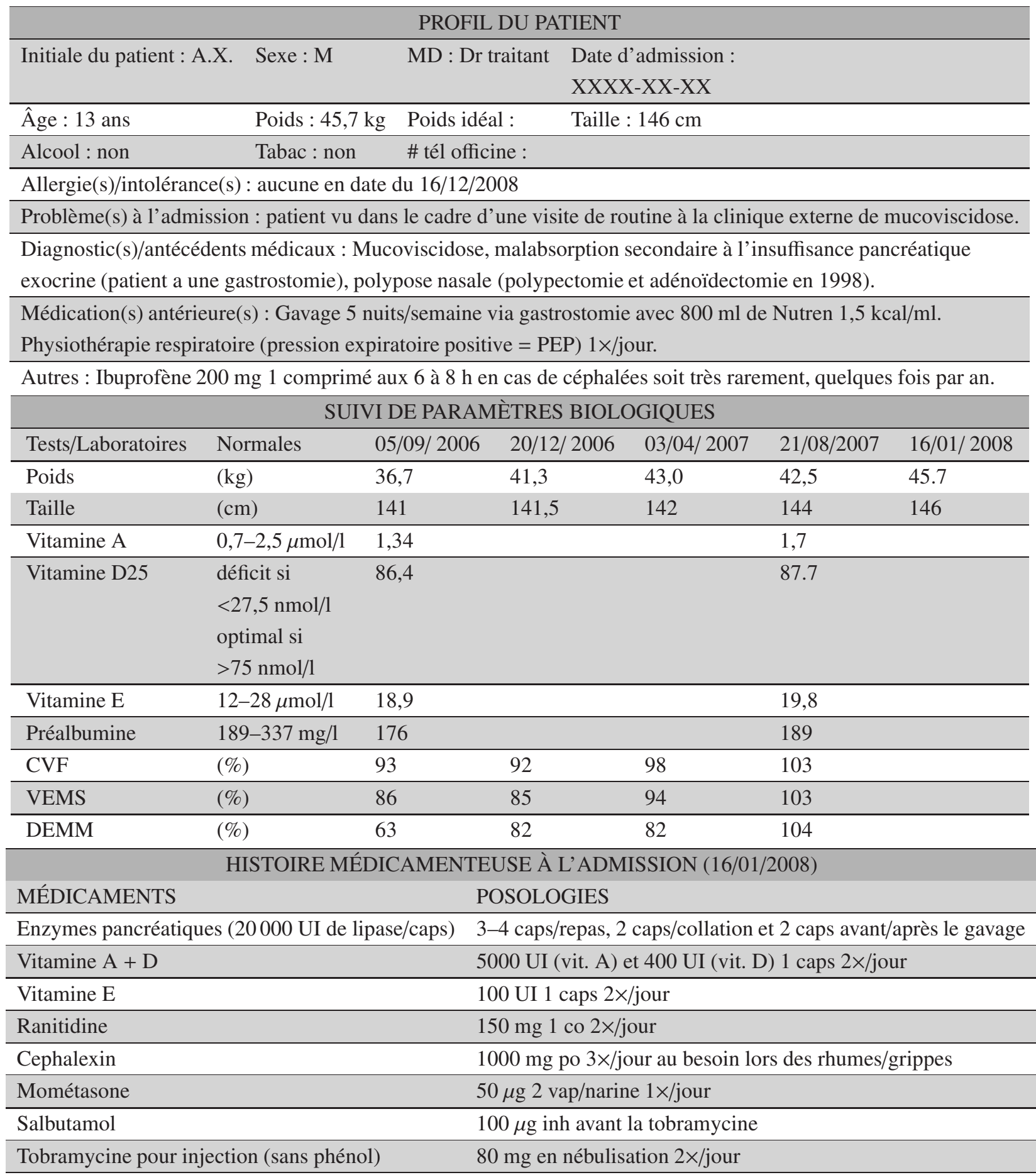


Tableau III. suite.

\begin{tabular}{|c|c|c|}
\hline \multicolumn{3}{|c|}{ AUTRES ÉLÉMENTS PERTINENTS } \\
\hline \multicolumn{3}{|c|}{$\begin{array}{l}\text { Dernières cultures : } \\
16 / 01 / 2008: \text { flore normale } \\
07 / 11 / 2007: S \text {. aureus sensible à tout et } P \text {. aeruginosa sensible à tout sat } \\
03 / 04 / 2007: S \text {. aureus sensible à tout et } P \text {. aeruginosa sensible à tout } \\
01 / 12 / 2006: S \text {. aureus } \text { sensible à tout }\end{array}$} \\
\hline $\begin{array}{ll}\text { Problème } & \text { 1) Colonisation à } \\
& \text { Pseudomonas aeruginosa } \\
& \text { (résistant au colistiméthat }\end{array}$ & $\begin{array}{l}\text { 2) Malabsorption secondaire à } \\
\text { l'insuffisance pancréatique } \\
\text { exocrine }\end{array}$ & $\begin{array}{l}\text { 3) Atteinte pulmonaire } \\
\text { chez patient atteint } \\
\text { de mucoviscidose }\end{array}$ \\
\hline $\begin{array}{ll}\text { Résultat(s) } & \text { Idéalement, éradiquer } \\
\text { recherché(s) } & \text { P. aeruginosa } \\
& \text { - Prévenir les surinfections } \\
& \text { pulmonaires à } P \text {. aerugino } \\
& \text { et la diminution potentiell } \\
& \text { irréversible des EFR } \\
& \text { (épreuve fonctionnelle } \\
& \text { respiratoire). } \\
& \text { - Éviter la résistance } \\
& \text { antibiotique. } \\
& \text { - Éviter les interactions et } \\
& \text { effets indésirables. } \\
\end{array}$ & $\begin{array}{ll} & \text { - Éviter la dénutrition et les } \\
\text { troubles de croissance. } \\
\text { s } \\
\text { sa } \\
\text { lement (Aider l'absorption des protéine } \\
\text { lipides et vitamines liposolubles } \\
\text { - Éviter les symptômes de } \\
\text { malabsorption (stéatorrhée, doul } \\
\text { abdominales...). } \\
\text { - Éviter les interactions et les eff } \\
\text { indésirables. } \\
\text { les }\end{array}$ & $\begin{array}{l}\text { - Favoriser l'expectoration des } \\
\text { sécrétions pulmonaires. } \\
\text { es, - Augmenter la pénétration et, } \\
\text { par conséquent, l'efficacité des } \\
\text { médicaments pris par } \\
\text { inhalation/nébulisation. } \\
\text { leurs - Maintenir les EFR. } \\
\text { - Prévenir les surinfections } \\
\text { fets } \\
\text { pulmonaires. } \\
\text { - Éviter les interactions et les effets } \\
\text { effets indésirables. }\end{array}$ \\
\hline $\begin{array}{l}\text { Solution(s) Tx suppressif chronique } \\
\text { possible(s) du P. aeruginosa } \\
\text { - Colistiméthate } 30-150 \mathrm{mg} \\
\text { en nébulisation } 2 \times / \text { jour, } \\
\text { - Tobramycine } 80-300 \mathrm{mg} \\
\text { en nébulisation } 2 \times / \text { jour } \\
\text { - Bronchodilatateur pour le } \\
\text { risque de bronchospasmes. }\end{array}$ & $\begin{array}{l}\text { 1) Enzymes pancréatiques. } \\
\text { 2) Vitamine A et D } 5000 \text { UI et } 400 \text { UI } \\
\text { 3) Vitamine E } 100 \text { UI. } \\
\text { 4) Vitamine K } 1-10 \mathrm{mg} 2 \times / \text { semaine } \\
\text { jusqu'à } 1 \times / \text { jour. } \\
\text { 5) Oméprazole ou lansoprazole. } \\
\text { 6) Ranitidine } 4-6 \mathrm{mg} / \mathrm{kg} / \mathrm{j} \\
\text { (max } 300 \mathrm{mg} / \mathrm{jour} \text { ). } \\
\text { 7) Diète hypercalorique (130-150\% } \\
\text { ANR, 35-40 \% lipides). } \\
\text { 8) Suppléments alimentaires gavages } \\
\text { ou nutrition parentérale. }\end{array}$ & $\begin{array}{l}\text { - Kiné respiratoire } 1 \times / \text { jour. } \\
\text { I.- Dornase alpha } 2,5 \mathrm{mg} \\
\text { en nébulisation } 1 \times / \text { jour. } \\
\rightarrow \text { rejeté, non requis par la condition } \\
\text { clinique actuelle. } \\
\text { - Solution hypertonique de } \mathrm{NaCl} \text { à } 7 \% \text {, } \\
\rightarrow \text { rejeté, moins pratique, pas de } \\
\text { formulation commerciale } \\
\text { - } \mathrm{N} \text {-acétylcystéine } 5-10 \mathrm{ml} \\
\text { de solution à } 10 \text { ou } 20 \% \\
\text { en nébulisation } 3 \text { à } 4 \times / \text { jour } \\
\rightarrow \text { rejeté, fréquence d'administra- } \\
\text { tion élevée et mauvaise odeur }\end{array}$ \\
\hline $\begin{array}{l}\text { Solution(s)Tobramycine } 80 \mathrm{mg} \text { en } \\
\text { retenue(s) nébulisation } 2 \times / \text { jour, } \\
\text { précédée de salbutamol } \\
100 \mu \mathrm{g} 2 \text { inhalations. }\end{array}$ & $\begin{array}{l}\text { 1) Enzymes pancréatiques : } \\
1750 \mathrm{UI} / \mathrm{kg} / \mathrm{repas} \\
\times 3,895 \mathrm{UI} / \mathrm{kg} / \text { collation } \\
\times 3,1750 \mathrm{UI} / \mathrm{kg} / \text { gavage } \\
\text { (9625 UI/kg/jour). } \\
\text { 2) Vitamine A } 5000 \text { UI et vitamine D } \\
400 \text { UI } 1 \text { caps } 2 \times / \text { jour. } \\
\text { 3) Vitamine E } 200 \text { UI } 1 \text { caps } 1 \times / \text { jour. } \\
\text { 6) Ranitidine } 150 \mathrm{mg} 2 \times / \text { jour } \\
\text { (6,6 mg/kg/jour). }\end{array}$ & $\begin{array}{l}\text { - Kiné respiratoire (PEP) } \\
1 \times / \text { jour (les matins du week- } \\
\text { end et les soirs de semaine). }\end{array}$ \\
\hline
\end{tabular}


Tableau III. suite.

\begin{tabular}{|c|c|c|c|}
\hline & & $\begin{array}{l}\text { 7) Diète hypercalorique (130-150\% } \\
\text { apports nutritionnels recommandés : }\end{array}$ & \\
\hline & & $\begin{array}{l}\text { 35-40 \% lipides). } \\
\text { 9) Gavage via gastrostomie avec } \\
800 \mathrm{ml} \text { de Nutren } 1,5 \mathrm{kcal} / \mathrm{ml} \text {, } \\
5 \text { nuits/semaine. }\end{array}$ & \\
\hline $\begin{array}{l}\text { Intervention(s) } \\
\text { à effectuer }\end{array}$ & $\begin{array}{l}\text { - Vérifier observance à } \\
\text { tobramycine et ordre } \\
\text { d'administration : } \\
\text { 1) salbutamol, 2) kiné } \\
\text { respiratoire, } \\
\text { 3) tobramycine. } \\
\text { - Vérifier la tolérance : } \\
\text { bronchospasmes? }\end{array}$ & $\begin{array}{l}\text { - Vérifier l'observance et la technique } \\
\text { de prise des enzymes } \\
\text { et vitamines (prises des enzymes } \\
\text { au début du repas). } \\
\text { - Vérifier si présence de symptômes } \\
\text { de malabsorption. }\end{array}$ & $\begin{array}{l}\text { - Si les EFR diminuent } \\
\text { ou si le patient commence à } \\
\text { avoir des sécrétions qu'il } \\
\text { ne peut expectorer, considérer } \\
\text { la possibilité d'ajouter } \\
\text { de la dornase alpha. }\end{array}$ \\
\hline $\begin{array}{l}\text { Suivi } \\
\text { (monitorage) } \\
\text { et } \\
\text { fréquence }\end{array}$ & $\begin{array}{l}\text { Efficacité : maintien des EFP, } \\
\text { éradication ou absence de } \\
\text { surinfection pulmonaire à } \\
P . \text { aeruginosa } \\
\text { Innocuité : bronchospasmes? } \\
\text { Suivi observance }\end{array}$ & $\begin{array}{l}\text { Efficacité : } \uparrow \text { taille/poids (courbes de } \\
\text { croissance), absence de symptômes de } \\
\text { malabsorption, dosage annuel des } \\
\text { vitamines ADE. } \\
\text { Innocuité : effets indésirables gastro- } \\
\text { intestinaux. } \\
\text { Observance lors des cliniques externes } \\
\text { (nutritionniste et/ou la pharmacienne). }\end{array}$ & $\begin{array}{l}\text { Efficacité : maintien des EFR. } \\
\text { Observance : vérifiée surtout } \\
\text { par l'inhalothérapeute lors } \\
\text { des cliniques externes. }\end{array}$ \\
\hline $\begin{array}{l}\text { Résultat(s) } \\
\text { obtenu(s) }\end{array}$ & $\begin{array}{l}\text { - Plan de prise des } \\
\text { médicaments ok } \\
\text { - Tolérance patient ok } \\
\text { - État pulmonaire stable }\end{array}$ & $\begin{array}{l}\text { Observance et technique de prise des } \\
\text { enzymes/vitamines adéquates (oubli } \\
\text { quelques fois/semaine). } \\
\text { Rappel de l'indication des vitamines au } \\
\text { patient. } \\
\text { - } \uparrow \text { poids ( } 50^{\mathrm{e}} \text { percentile), } \uparrow \text { suboptimale } \\
\text { pour taille }\left(10^{\mathrm{e}} \text { percentile). }\right. \\
\text { - Dosages des vitamines ADE adéquats, } \\
\text { préalbumine à la limite inférieure de la } \\
\text { normale. }\end{array}$ & $\begin{array}{l}\text { - Pt connu comme très } \\
\text { observant à sa kiné } \\
\text { respiratoire. } \\
\text { - Les EFR sont stables et } \\
\text { le patient va bien sur le plan } \\
\text { respiratoire. }\end{array}$ \\
\hline
\end{tabular}

le concept depuis plusieurs années, ce concept est encore peu enseigné en France. Cet enseignement en France est rarement effectué par un praticien de terrain qui côtoie des patients au quotidien. L'utilisation du terme soins pharmaceutiques est largement utilisée dans la documentation scientifique nordaméricaine alors qu'elle émerge en France. Le pharmacien est-il un soignant? Le pharmacien doit-il assumer une responsabilité quant aux résultats cliniques recherchés et aux solutions proposées? Nous pensons qu'il doit jouer ce rôle, alors que la pharmacothérapie ne cesse de se complexifier.
Cette étude pilote indique qu'il est faisable, par le biais d'un outil de plan de soins pharmaceutiques, d'exposer des étudiants à ce modèle de pratique pharmaceutique. Bien que les étudiants français aient reçu une exposition minimale au concept et à l'outil par le biais d'un guide de formation, tous les étudiants ont été en mesure d'utiliser minimalement l'outil. Toutefois, le temps passé à la collecte des données par les étudiants français pour rédiger le plan de soins était plus important (écart d'au moins huit heures) que pour les étudiants du groupe québécois. Le troisième étudiant français, 
qui n'avait jamais été exposé à la pratique hospitalière, n'y a passé que deux heures et a délivré un travail très incomplet. La dernière étudiante française se rapproche de la moyenne québécoise vraisemblablement grâce à l'expérience déjà acquise. Ainsi le manque d'exposition à la pratique pourrait expliquer en partie pourquoi les étudiants français n'ont pas été en mesure de fournir autant de données sur le patient, sur les problèmes reliés à la pharmacothérapie, sur les solutions et les suivis à mettre en place.

De plus, notre étude pilote montre un niveau d'accord similaire à la plupart des énoncés retenus pour évaluer l'utilité et l'applicabilité de l'outil. Les remarques des étudiants français sont particulièrement intéressantes : elles expriment un besoin plus fort que les étudiants québécois. Elles pourraient refléter les différences de curriculum des études pharmaceutiques entre les deux pays. En France, l'enseignement est encore très centré sur la connaissance des traitements et beaucoup moins sur la méthode d'analyse du traitement, telle qu'elle est nécessaire pour le plan de soins pharmaceutiques. Le plan de soins proposé dans le cadre de l'étude a permis d'accompagner et de guider dans cet acte les étudiants français. Les étudiants français souhaitent que des espaces additionnels soient ajoutés pour documenter certains aspects, notamment les conditions de délivrance et d'administration. Alors que les étudiants français sont enthousiastes à l'idée d'utiliser un outil détaillé dans leur pratique professionnelle, les étudiants québécois, qui ont rédigé plusieurs plans de soins au cours de leur formation, sont davantage critiques quant à l'utilité d'un tel outil au quotidien. Cette différence d'appréciation ne nous étonne pas. À notre avis, l'outil de plan de soins pharmaceutiques proposé enseigne une démarche systématique aux étudiants, qui peuvent reproduire la démarche sans forcément utiliser le même outil dans leur pratique professionnelle. Il faut aussi souligner que la documentation des interventions pharmaceutiques peut être améliorée, en France comme au Québec, et que cet outil peut encourager une telle documentation et un partage du plan de traitement avec les autres professionnels.
Au niveau de la simulation et des verbatim recueillis, il est intéressant de noter que les questions posées et les rétroactions données par le pharmacien du groupe Québec s'adressent davantage à l'étudiant en situation de résolution de problèmes tandis que le pharmacien du groupe France recourt davantage à des questions qui ne sont pas forcément spécifiques à ce patient et cet étudiant. L'approche de soins pharmaceutiques vise à personnaliser l'intervention et le suivi dans un contexte clinique précis et non dans un contexte générique applicable à un groupe de patients similaires. Ces différences expriment sans doute le décalage de la pratique entre les deux pays. Ceci est sans doute révélateur du développement différent des soins pharmaceutiques dans les deux pays et au fait que les enseignants québécois sont en grande majorité praticiens dans un service clinique, ce qui n'est pas le cas à l'hôpital Robert Debré.

Peu d'études ont évalué l'utilité et l'applicabilité d'outils de plans de soins pharmaceutiques. Isetts décrit un projet d'évaluation de la capacité d'étudiants en formation à prodiguer des soins pharmaceutiques. Réalisé auprès de 14 étudiants durant un semestre, les étudiants ont été en mesure d'identifier 61 problèmes reliés à la pharmacothérapie chez 18 patients ${ }^{[15]}$. Chisholm et al. ont évalué chez un groupe de 21 étudiants un modèle d'apprentissage expérientiel et pyramidal ${ }^{[16]}$. Des étudiants de troisième année étaient invités à évaluer des patients dans dix secteurs cliniques et à présenter leurs plans de soins à un groupe de sept étudiants et un professeur. De plus, des étudiants de quatrième année en pharmacie étaient invités à assister les étudiants de troisième année au cours de leur démarche. Cette démarche pyramidale a permis de réduire de 300 à 80 heures la contribution professorale en mettant davantage à contribution les étudiants. De façon différente, notre étude suggère qu'un jumelage d'étudiants France-Québec, notamment par le biais de visioconférence, peut contribuer à la formation au même titre que les étudiants de quatrième année dans le projet de Chisholm. Cette expérience a été particulièrement stimulante pour les étudiants français qui ont pu se mesurer à des étudiants dont 
l'esprit est «théoriquement » mieux formé aux plans de soins pharmaceutiques. L'apport des nouvelles technologies, telle que la visioconférence, est certainement une solution convaincante pour enseigner et provoquer l'émulation. C'est une voie à encourager pour les facultés ou autres hôpitaux qui n'ont pas ou peu d'enseignant-praticiens de pharmacie clinique.

Depuis quelques années, de nombreux organismes de prestation sécuritaire de soins recommandent que soit implanté un bilan comparatif de médicaments à l'admission et à la sortie du patient en établissement de santé ${ }^{[17]}$. Plus qu'une simple histoire médicamenteuse à l'arrivée, le bilan comparatif vise à documenter l'ensemble des antécédents médicaux et pharmaceutiques du patient, à signifier clairement ce qui doit être cessé, modifié et poursuivi durant l'hospitalisation. De même, à la sortie du patient, l'équipe traitante, avec la collaboration du pharmacien, doit produire un bilan comparatif de départ, qui indique clairement au pharmacien de ville ce qui doit être cessé, poursuivi, modifié ou initié. Le concept de plan de soins pharmaceutiques, qui peut n'être qu'une démarche académique, s'intègre tout à fait dans la démarche de bilan comparatif à l'arrivée et au départ, en présentant non seulement les ordonnances mais aussi les objectifs visés.

Cette étude comporte des limites. Il s'agit d'une étude pilote observationnelle. Bien que les résultats suggèrent qu'il soit faisable et simple d'implanter un outil de plan de soins pharmaceutiques en France, la taille limitée de la cohorte de pharmaciens et d'étudiants impliqués ne permet pas de généraliser les résultats à un plus grand groupe. La grille utilisée pour évaluer l'impact de l'outil n'a pas fait l'objet d'une validation externe. Enfin, l'étude comporte un biais d'observation compte tenu que les étudiants recrutés étaient conscients de la comparaison et des interactions par visioconférence.

\section{Conclusion}

Il existe peu de données sur les outils utilisés en support aux soins pharmaceutiques. Cette étude pilote suggère qu'un outil de plan de soins pharma- ceutiques utilisé au Québec pourrait aussi être utilisé en France afin de promouvoir la philosophie des soins pharmaceutiques auprès des étudiants français. Il met en évidence combien les échanges par le travail en réseau grâce aux nouvelles technologies de communication peuvent générer la stimulation entre étudiants dans le cadre de l'enseignement et de la formation.

\section{Remerciements}

Nous aimerions remercier les étudiants cinquième année hospitalo-universitaire de la faculté de pharmacie de l'université Paris V-René Descartes, Sophie, Estelle, Mouna et Glenn, ainsi que les étudiants de premier cycle de la faculté de pharmacie de l'université de Montréal, Michael, Catherine, Brian et Josiane, pour leur enthousiasme et participation active au projet.

\section{Contributions}

Quatre pharmaciens tuteurs ont construit, conduit et animé le projet : Jean-François Bussières qui est le coordinateur et est à l'initiative du projet, Ariane Blanc, Annie Lavoie et André Rieutord. Le plan de soins présenté dans le tableau III est une contribution de Josiane Gagnon-Bagheri, étudiante participant au projet.

\section{Références}

1. Hepler CD, Strand LM. Opportunities and responsibilities in pharmaceutical care. Am J Hosp Pharm 1990;47:533-43.

2. Fédération internationale pharmaceutique. Déclarations de la FIP sur les normes professionnelles - les soins pharmaceutiques. [On-line] Disponible sur http://www . fip.org/www2/uploads/database_ file.php?id=270\&table_id=site (visité le 200805-10).

3. Jones EJ, Mackinnon NJ, Tsuyuki RT. Pharmaceutical care in community pharmacies: practice and research in Canada. Ann Pharmacother 2005;39:1527-33. 
4. Christensen DB, Farris KB. Pharmaceutical care in community pharmacies: practice and research in the US. Ann Pharmacother 2006;40:1400-6.

5. Noyce PR. Providing patient care through community pharmacies in the UK: policy, practice, and research. Ann Pharmacother 2007;41:861-8.

6. Eickhoff C, Schulz M. Pharmaceutical care in community pharmacies: practice and research in Germany. Ann Pharmacother 2006;40:729-35.

7. Krahenbuhl JM, Kremer B, Guignard B, Bugnon O. Pratical evaluation of the drug-related problem management process in Swiss community pharmacies. Pharm World Sci 2008 DOI:10.1007/s11096-008-9217-4

8. Cradock JC, Whitfield GR, Menzie JW, Fortner CL. Postadmission drug and allergy histories recorded by a pharmacist. Am J Hosp Pharm 1972;29:250-2.

9. Cooper CR. Techniques for the selection and monitoring of patients by pharmacists. Am J Hosp Pharm 1972;29:334-8.

10. Bennett RW, Bryant BG, Kelly AL, Pruett JD. Development of a pharmacy care plan. Am J Hosp Pharm 1973;30:698-701.

11. Chase PA, Bainbridge J. Care plan for documenting pharmacist activities. Am J Hosp Pharm 1993;50:1885-8.

12. Lapane KL, Hiris J, Hughes CM, Feinberg J. Development and implementation of pharmaceutical care planning software for nursing homes based on the Fleetwood Model. Am J Health Syst Pharm 2006;63:2483-7.

13. Canaday BR, Yarborough PC. Documenting pharmaceutical care: creating a standard. Ann Pharmacother 1994;28:1292-6.

14. Rich DS. JCAHO's pharmaceutical care plan requirements. Hosp Pharm 1995;30:315-9.

15. Isetts BJ. Evaluation of pharmacy student's abilities to provide pharmaceutical care. Am J Pharm Edu 1999;53:11-20.

16. Chisholm MA, DiPiro JT, Fagan SC. Instructional design and assessment. An innovative introductory pharmacy practice experience model. Am J Pharm Edu 2003;67:171-8.

17. The Lewin Group. Medication therapy management services: a critical review. J Am Pharm Assoc 2005;45:580-7.

Correspondance et offprints : Jean-François Bussières, Département de Pharmacie, CHU Ste-Justine 3175 Côte Ste-Catherine Montréal (Québec) H3T 1C5 Canada. Téléphone : (514) 345-4931 (5053) Fax : (514) 345-4972

Mailto : jf.bussieres@ssss.gouv.qc.ca 